\title{
Performance Characteristics of the 60 kA SMES Conductor
}

\author{
J.M. Pfotenhauer, M.K. Abdelsalam, O.D. Lokken, Z. Jiang, J.S. Waldrop, and S. Yang
} Applied Superconductivity Center, University of Wisconsin - Madison, Madison. WI 53706

\author{
O.R. Christianson, E.F. Daly, J.E. King, and D. Pavlik \\ Westinghouse STC, Pittsburgh, PA 15235 \\ E. Brogren and D. DeGraaf \\ Chicago Bridge \& Iron, Plainfield, IL 60544 \\ F. Kessler and A. Ludbrook \\ Ebasco Services, Inc. Madison, WI 53713
}

\begin{abstract}
We report the operating characteristics of the monolithic SMES conductor designed for operation at $60 \mathrm{kA}, 5.1$ tesla in a $1.8 \mathrm{~K}$ bath of subcooled helium. Details of the experimental arrangement and procedures are presented as background. At $1.85 \mathrm{~K}$ the conductor has been operated up to $75 \mathrm{kA}$, and with maximum field conditions of 4.25 tesla. Conductor performance is compared to the load line and design point of the Engineering Test Model (ETM). Transient stability is also demonstrated. Maximum conditions experienced by the conductor were limited by facility operation rather than by the conductor itself.
\end{abstract}

\section{INTRODUCTION}

During the summer of 1990 , the SMES-ETM Proof of Principle Experiment (POPE) produced data validating the dynamic stability theory which governs much of the design for the $60 \mathrm{kA}$ SMES conductor [1]. Specifically, good agreement is displayed between the theory and experiment regarding the existence and magnitude of a threshold current, fast normal zone propagation velocities and the phenomenon of finite length traveling normal zones. While the data were gathered in temperature and field conditions different from those of the SMES-ETM, extrapolation of the results guaranteed that the conductor could operate in SMES-ETM conditions without the possibility of normal zone instabilities. The data also suggested that the conductor could operate reliably at a higher current and field than the earlier design point of $50 \mathrm{kA}$ and 4 tesla. Thus, efforts were directed at demonstrating reliable operation at a new design point of $60 \mathrm{kA}$ and 5.1 tesla. The operating environment for the superconductor is a bath of subcooled superfluid helium at 1.8 K.

Preparations for operating the coil at the design point

Manuscript received October 18, 1994

This work was supported by the Defense Nuclear Agency under contract \# DNA001-92-C-0064. included significant upgrading of three major systems from their 1990 condition. In particular, the vacuum integrity of the dewar was improved to a $10^{-5} \mathrm{~Pa}$ pressure, the capacity of the superfluid subcooler refrigeration system was increased to 45 watts at $1.8 \mathrm{~K}$, and the protection system for the background magnet was developed to a much higher sophistication level in keeping with the method described in Hilal et al [2].

Additionally, during September 1993 a complete conductor splice - both superconductor and stabilizer - was inserted into the test coil and subsequently tested. Details of the splice and its performance are reported in a companion paper [3]. The final operation of the POPE occurred between October 28 and November 13, 1993.

\section{EXPERIMENTAL ARRANGEMENT AND PROCEDURES}

Configuration: As has been described in detail elsewhere [4], the Proof of Principle Experiment is designed to reproduce in a laboratory experiment, the identical conditions for the conductor as would be realized in the $20 \mathrm{MWh}$ SMESETM design. The experiment incorporates a 3 turn test coil of the one inch diameter monolithic conductor wound with a 1 meter bore and supported by a high strength (6061-T6 and 7075-T6) aluminum structure. DC current is provided to the test coil from a $100 \mathrm{kA}$ power supply and through a pair of $75 \mathrm{kA}$ current leads. As the 3 turn test coil produces a self field of only 1.8 tesla at $60 \mathrm{kA}$, the additional background field was to be supplied by a split pair superconducting magnet configured with the two halves located immediately above and below the test coil. Difficulties in the operation of the background magnet proved to be the limiting factor in the tests rather than any performance characteristic of the test coil.

Instrumentation on the test coil is comprised of 5 identical conductor disturbance heaters (each of $22.2 \mathrm{~cm}$ length) at various locations, thermometers mounted inside the conductor, hall probes mounted at three different locations around the coil, and voltage taps encompassing the entire test 
coil circuit - that is, from the top of the $(+)$ current lead to the top of the (-) current lead. The voltage taps encompassing the entire test coil circuit were also used as input for the test coil protection system.

The combined test coil and background magnet assembly sits in a liquid helium vessel which is equipped with a liquid helium based JT refrigeration system so that the magnet and test coil operated in a subcooled superfluid helium bath at 1.8 $\mathrm{K}$ and $101 \mathrm{kPa}$. Thermal insulation for the helium vessel is provided by virtue of being located inside a larger vacuum vessel, and maintaining a vacuum of better than $10^{-4} \mathrm{~Pa}$.

Operating Procedure: The primary goal of the last run of the the POPE was to verify operation of the conductor at its design point. In keeping with this goal, the operating procedures for running the test were developed to provide the most direct approach at achieving that design point. Contigent on that achievement, and as allowed by time, equipment and funding, additional tests were planned to measure stability characteristics of the conductor in the ETM operating conditions. The procedural sequence is as follows:

After verifying the integrity of all circuits and vacuum spaces, the test coil and background magnet were cooled from room temperature to $4.2 \mathrm{~K}$ solely by introducing cold helium gas through a sparging ring at the bottom of the helium vessel. Although this cooldown procedure required additional helium as compared to a precool operation with liquid nitrogen, it was determined to have an economic advantage due to a significant reduction in the associated man-hours. Once the helium vessel was filled with liquid helium, the JT refrigeration system was activated and the test coil and magnet were cooled to $1.85 \mathrm{~K}$.

System shakedown procedures were conducted at this point to verify operation of the conductor splice and to measure the coupling coefficients between the two halfs of the background magnet and the test coil. These last measurements are required for operation of the background magnet protection system [2]. During verification of the conductor splice, it was observed that the associated heat load from operating the test coil at $60 \mathrm{kA}$ did not permit the refrigeration system to maintain the $1.85 \mathrm{~K}$ operating temperature. Subsequent analysis of the conductor splice resistance [3] revealed that it caused an additional 10 watt heat load and was responsible for the gradual warming. Thus, although the splice was demonstrated as fully satisfying ETM requirements, the effect on the POPE operation determined that in order to maintain the bath operating temperature the most direct approach to the design point required steady state operation of the background magnet with a subsequent ramp-up, ramp-down cycle of the test coil to $60 \mathrm{kA}$.

\section{DESIGN POINT ACHIEVEMENTS}

Combined Coil Ramping: The adopted approach to the combined current, field design point of $60 \mathrm{kA}$ and 5.1 tesla is displayed in figure 1 . In the first step the background magnet was charged to $330 \mathrm{~A}$ and the test coil was cycled to $60 \mathrm{kA}$ resuling in a total field of 2.98 tesla. The magnet current was then increased to $675 \mathrm{~A}$ and the test coil ramp to $60 \mathrm{kA}$ was again initiated. At a test coil current of $55.5 \mathrm{kA}$ a voltage signal in the background magnet tripped its protection system and forced a dump of the magnet energy. Most of the magnet stored energy was deposited in a room temperature dump resistor, but a significant amount was also dissipated in the helium bath. The trip of the magnet protection system induced a subsequent trip ( 0.05 seconds later ) of the test coil protection circuit and a controlled ramp-down of the test coil followed.

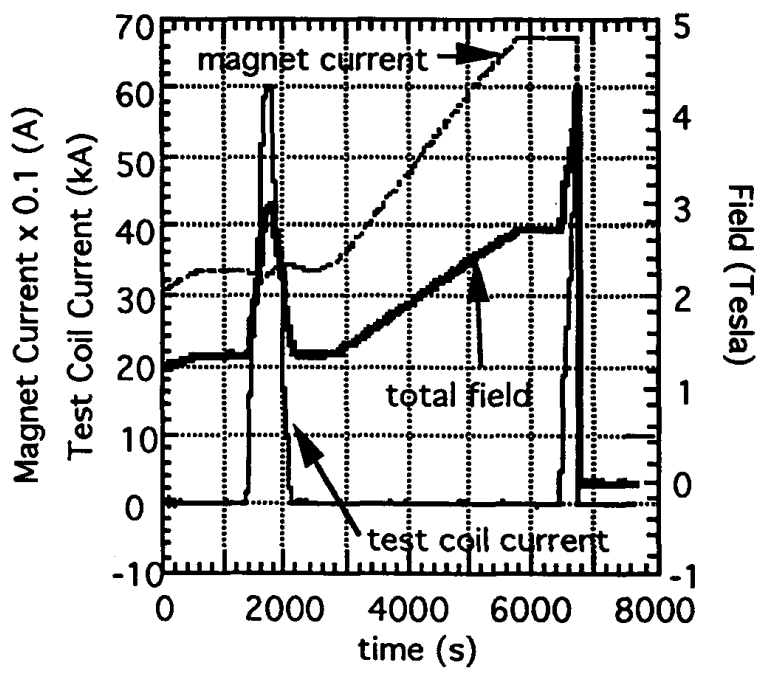

Figure 1. Combined coil operation

The maximum combined current and field conditions experienced by the coil in this test were $55.5 \mathrm{kA}$ and 4.25 tesla. The value of the total magnetic field has been obtained from an ANSYS finite element analysis and represents the maximum combined background field and self field of the strands in the test conductor. It should be noted that these values are in excess of the original design point for this conductor of $50 \mathrm{kA}$ and 4 tesla. Subsequent measurements of the background magnet inductance (and mutual coupling coefficients) indicated that it experienced damage during the energy dump; no further operation of the magnet was pursued.

Independent Test Coil Ramp: In the $75 \mathrm{kA}$ test, a linear steady current ramping was achieved by utilizing the automatic current ramping feature of the $100 \mathrm{kA}$ power supply. In this test the current limit was set to $75 \mathrm{kA}$, and the ramp time to 300 seconds. Figure 2 displays a record of this test. The conductor successfully reached the set point of $75 \mathrm{ka}$ with zero resistance in the test coil. A resistance of 2.5 $\mathrm{n} \Omega$ was observed across the new conductor splice (electrically between the + side of the test coil and the + current lead), and a larger $2.6 \mu \Omega$ resistance was observed across the transition 
between the (-) side of the test coil and the (-) current lead. It should be noted here that the conductor displayed no indications that it could not be operated at higher currents. The $75 \mathrm{kA}$ limit was adopted in keeping with the manufacturer's design current for the current leads and due to concern over the voltage established at the transition near the $(-)$ current lead.

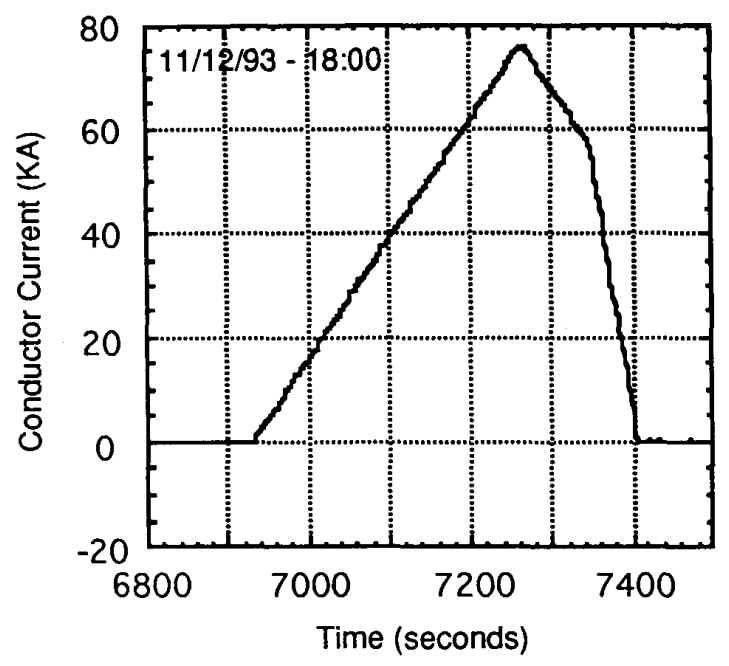

Figure 2. Independent ramp of test coil to $75 \mathrm{kA}$

Conductor Operation Summary: Figure 3 displays a summary of the operating achievements for the $60 \mathrm{kA} \mathrm{SMES}$ conductor. Use of Morgan's correlation [5] provides the projection of the above results onto the ETM operation load line and demonstrates that the conductor has been operated to conditions equivalent to $89 \%$ of the ETM design point. Aside from the equipment difficulties, there are no indications that the conductor can not be operated at $100 \%$ of the ETM design point as it has been designed.

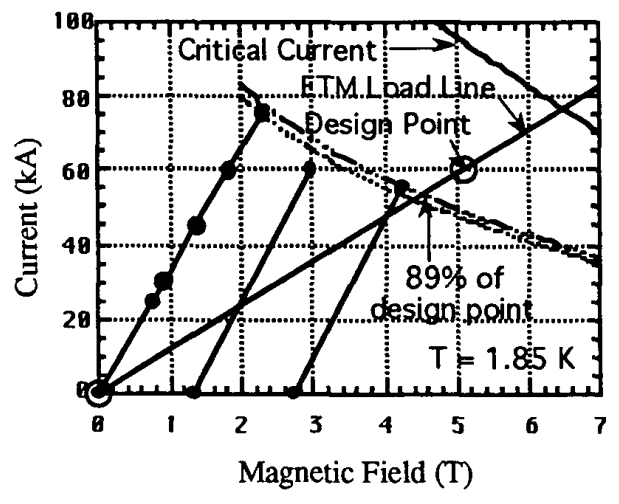

Figure 3. Summary of conductor performance

\section{STABILITY TESTS AND MEASUREMENTS}

Conductor stability measurements have been severely limited during the POPE runs in 1993. These were primarily limited by the operation of the background magnet, but also through limitations of the vapor cooled leads. The sensitivity of the background magnet to test coil ramping which was experienced during the design point efforts, and the significant resultant energy deposition in the helium bath when the background magnet protection system tripped prohibited further testing with the background magnet. Furthermore, calculations of the threshold current for dynamic stability in zero field at $1.9 \mathrm{~K}$ are well in excess of $75 \mathrm{kA}$ and were therefore not pursued.

Transient stability measurements have been undertaken with the bath temperature in the superfluid range at $1.9 \mathrm{~K}$, a test coil current of $60 \mathrm{kA}$, and with zero background magnetic field. In this test, the $2 \mathrm{~m} \Omega$ heater in the center turn of the test coil was used to drive the conductor normal. Increasing levels of energy were deposited in the heater, and at the maximum available energy pulse from the pulsed power supply ( 500 Joules dissipated in the heater) a normal zone was created in the conductor. Figure 4 displays the normal zone voltage and the conductor temperature in the center of the heated zone, and demonstrates that the conductor recovers from this disturbance.

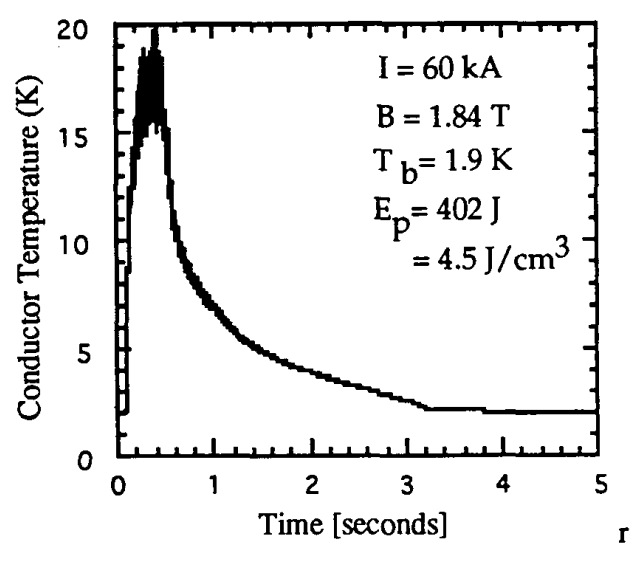

Figure 4. Transient response of SMES-ETM conductor.

Modelling efforts have been carried out to quantify the percentage of heat dissipated in the heater which is transferred to the conductor, and the corresponding percentage of heat transferred directly to the nearby liquid helium. Of the $470 \mathrm{~J}$ dissipated by the heater, $402 \mathrm{~J}$ or $85 \%$ are involved in heating up the conductor. This is equivalent to $1826 \mathrm{~J} / \mathrm{m}$, or 4.5 $\mathrm{J} / \mathrm{cm}^{3}$ Furthermore, we have learned from modelling the transient and dynamic stability of the conductor, that the stability boundary is somewhat insensitive to the pulse length of thermal disturbances (between a $5 \mathrm{msec}$ and the 250 
msec puises which our heater produces) provided they deliver the same total energy to the conductor. This may be understood by recognizing that heat transfered into the superfluid helium across a $\mathrm{He}$ I - He II phase boundary has a time dependence which is determined soley by the geometry of the bath. Thus, the cooling influences of the helium are identical for any conductor temperatures which drive the temperature of the helium immediately adjacent to the conductor above $T_{\lambda}$.

The above observations allow an meaningful extrapolation to be made regarding the transient stability of thge conductor at the ETM design point. Modelling results predict a $13 \%$ decrease in the allowable disturbance energy if the field is increased to 5.1 tesla. Thus it is expected that the SMESETM conductor will be transiently stable against energy disturbances at least up to $350 \mathrm{~J}\left(3.9 \mathrm{~J} / \mathrm{cm}^{3}\right)$.

The role of the superfluid helium bath in providing the extreme stability of this conductor merits attention. In particular, in the course of a 3 second duration stability process one dimensional thermal diffusion into a superfluid bath can produce a $50 \mathrm{mK}$ temperature rise as far away as 0.8 meters. For comparison, thermal diffusion in $4.2 \mathrm{~K}$ helium during the same time will propagate only $0.8 \mathrm{~mm}$. In view of this rapid propagation and absorption of heat by the superfluid helium, it is apparent that the best advantage is taken of those properties for cooling of high current composite superconductors in the open bath configuraton.

\section{SUMMARY}

Experiments carried out on the SMES-ETM conductor following the stability model verification tests made in 1990 have demonstrated stable operation beyond the earlier design point of $50 \mathrm{kA}$ and 4 tesla. The conductor has been operated at a maximum combined DC current and field of $55.5 \mathrm{kA}$ and 4.25 tesla, and at a maximum current of $75 \mathrm{kA}$ in its own self field of 2.30 tesla. Limitations in the investigation of conductor performance resulted from difficulties with the support equipment, and were not evident in the conductor itself. The maximum operating conditions achieved in this test represent a point on the ETM load line which is at $89 \%$ of the $60 \mathrm{kA}, 5.1$ tesla design point. The stability limitations at the design point are such that no traveling normal zones are possible, and the conductor is transiently stable against disturbances up to at least $350 \mathrm{~J}$ which is equivalent to $1590 \mathrm{~J} / \mathrm{m}$, or $3.9 \mathrm{~J} / \mathrm{cm}^{3}$.

\section{REFERENCES}

[1] Pfotenhauer, J.M., Abdelsalam, M.K., Bodker, F., Huttleston, D., Jiang, Z., Lokken, O.D., Scherbarth, D., Tao, B., and Yu, D., "Test Results from the SMES Proof of Principle Experiment," IEEE Trans on Magnetics MAG-27 (2), 17041707 (1991).

[2] Hilal, M.A., Vescey, G., Pfotenhauer, J.M., and Kessler, F., "Quench Detection of Multiple Magnet System," IEEE Trans. on Applied Superconductivity 4 (3), 109-114 (1994).

[3] D.W. Scherbarth, O.R. Christianson, E.F. Daly, T Kupiszewski, G.T. Mallick, D. Marschik, J.M. Pfotenhauer, M.K. Abdelsalam, O.D. Lokken, J.S. Waldrop, "Conductor Joint Performance During the Ebasco Team SMES POPE (Proof of Principle Experiment)" paper XXX presented at the 1994 Applied Superconductivity Conference, Boston, MA.

[4] Pfotenhauer, J.M., Bodker, F., Jiang, Z., Lokken, O.D., Tao, B., Yu, D., McIntosh, G.E., and Nickels, L.E., "A $100 \mathrm{kA}$, He II Cooled Conductor Test Facility," in Advances in Cryogenic Engineering, Vol. 37A, R.W. Fost, ed., Plenum Press, New York; 155-162 (1991).

[5] G. Morgan, "A Comparison of Two Analytical Forms for $\mathrm{J}_{\mathrm{c}}(\mathrm{B}, \mathrm{T})$ Surface," SSC Technical Note \# 310-1, Jan. 6, 1989. 